\title{
Tracking Trends in Marginalization and Deprivation Across Ontario with SDOH Mapper
}

\author{
Kieran Moore and Paul Belanger
}

KFL\&A Public Health, Kingston, ON, Canada

\section{Objective}

To describe how the Social Determinants of Health (SDOH) Mapper is used by KFL\&A Public Health to enhance real-time situational awareness of vulnerable populations across Ontario by facilitating the inclusion of information relating to marginalization and deprivation indices.

\section{Introduction}

Geographic Information System (GIS) technology provides visual tools, through the creation of computerized maps, graphs, and tables of geographic data, which can assist with problem solving and inform decision-making. One of the GIS tools being developed by KFL\&A Public Health is the Social Determinants of Health (SDOH) Mapper. The SDOH Mapper consists of layers of information related to deprivation and marginalization indices across Ontario. The SDOH Mapper facilitates the inclusion of information related to vulnerable populations with the use of both age and social determinants of health data into the GIS portal. This is useful for observing trends in marginalization and deprivation across dissemination areas in Ontario, and for examining health inequities in an area over time. The SDOH mapper will, in this way, improve knowledge transmission on the effects of poverty and marginalization on outcomes.

\section{Methods}

The SDOH Mapper allows users to customize maps demonstrating social determinants of health as they apply to specific geographic areas within the province of Ontario. The tool allows the user to visualize graphs with access to seven layers related to the marginalization and deprivation levels for a specified population so that they may better understand the health outcomes related to poverty, and thereby make informed decisions relating to health equity.

Deprivation layers are derived from the Deprivation Index of Health developed by the Quebec Public Health Institute (INSPQ). The deprivation social and material deprivation, as well as material and social deprivation combined. Marginalization data is obtained from the Ontario Marginalization Index (ON-Marg) developed by the Centre for Research on Inner City Health. The marginalization layers include residential instability, material deprivation, dependency, and ethnic concentration, which are derived from 18 indicators reflecting inequality and marginalization in Canada from both the 2001 and 2006 Census of Canada.

The SDOH Mapper encompasses a number of different functions and tools. Users can choose from several basemaps that identify different geographic features, such as topography, streets, or public venues, to best meet their needs. Users can apply seven deprivation and marginalization layers to the selected basemap, which allows for the appropriate visualization of social determinants of health as they apply to a specific geographic area. A legend is displayed that identifies the colour-coded quintiles for the marginalization and deprivation layers. Using the population summary tool, users are able to visualize graphs demonstrating the marginalization and deprivation layers for specific populations. Users can also measure the distance between two locations, as well as the perimeter and area of specified geographic locations using the draw and measure function.

\section{Conclusions}

The Public Health Informatics Team at KFL\&A Public Health is working to develop, in an ongoing systematic manner, a single access point to the GIS portal that will visualize multiple environmental and population based data sets in real-time. The single GIS portal will include the SDOH Mapper along with two other GIS applications developed by KFL\&A Public Health: the Public Health Information Management System (PHIMS) and the Tobacco Module. By providing available real-time data from multiple partners into the GIS portal, the Public Health Informatics team intends to assist with identifying health events earlier than traditional public health methods. Including the SDOH Mapper in the GIS portal provides an important social determinants of health lens through which environmental data, underlying population based indicators, and health events of interest can be examined and visualized, to provide users with an understanding of the effects of poverty on health outcomes

\section{Keywords}

poverty; marginalization; deprivation; map; GIS 